Acta Manilana 64 (2016), pp. 33-39

Printed in the Philippines

ISSN: 0065-1370

\title{
Enantioselective chiral 2-pyridyl-2-imidazoline organocatalyzed Aldol reactions in brine
}

\author{
Aileen Marice R. Baltazar ${ }^{1}$, Simon Budde ${ }^{2}$,Christian Faderl ${ }^{2}$, Aldrick B. Verano', \\ $\mathcal{E}$ Allan Patrick G. Macabeo*1
}

${ }^{1}$ Laboratory for Organic Reactivity, Discovery and Synthesis (LORDS), Research Center for the

Natural and Applied Sciences, University of Santo Tomas, 1015 Manila

${ }^{2}$ Institut für Organische Chemie, Universität Regensburg, Universitätsstrasse 31

D-93053 Regensburg, Germany

\begin{abstract}
Among the known organocatalyst manifolds, the organocatalytic utility of 2pyridylimidazolines has been less explored. In this study, a collection of chiral 2-pyridyl-2-imidazolines (picolinylimidazolines) were screened for organocatalytic activity towards enantioselective direct Aldol reaction. The chiral imidazolines (1a-1d) were prepared through conventional iodine-promoted oxidative condensation and cyclization of 2picolinaldehydes 2 (and/or 2,6-pyridine dicarbaldehyde) with chiral 1,2-diamine derivatives 3 in excellent yields ( $>96 \%$ isolated yields). Organocatalytic parameters were optimized to determine the best Aldol reaction conditions that would induce enantioselectivity such as catalyst loading, temperature, reaction time and solvent. Thus, using a $C_{2}$-symmetric cyclohexane-based 2-pyridyl-2-imidazoline organocatalyst 1c, the best conditions that gave excellent enantioinduction of up to 99:1 enantiomeric ratio (er) and yield were observed with brine as the solvent, a 1-h reaction period at room temperature and $10 \mathrm{~mol} \%$ as the minimum catalyst load. The reaction conditions were also amenable to a variety of benzaldehyde substrates with electron-donating and electron-withdrawing substituents. Our study demonstrates for the first time the asymmetric construction of $\beta$-hydroxy carbonyl structures using chiral $C_{2}$-symmetric 2-pyridyl-2-imidazolines as organocatalysts.
\end{abstract}

Keywords: Aldol reaction, organocatalysis, picolinylimidazoline, $\beta$-hydroxy carbonyl, asymmetric synthesis

\section{INTRODUCTION}

The design of a new catalyst for asymmetric reactions is one of the key challenges and a highly important task in organic synthesis. In the design of a new catalyst scaffold, molecular symmetry plays a powerful guideline as it

*To whom correspondence should be addressed allan.patrick.macabeo@ust.edu.ph /

allanpatrick_m@yahoo.com simplifies transition states and reduces the number of diastereomeric reaction sites to favorably effect high enantioselectivity [1]. Asymmetric organocatalysis has become a very rapidly growing field as a result of both the novelty of the concept and the selectivities attained by many organocatalytic transformations [2]. Among the main advantages of this methodology is the stability of intermediate species participating in the catalytic 
cycle toward water and oxygen and therefore reactions do not require special care with regard to the use of inert atmosphere or dry solvent [3]. In addition, the presence of hazardous metals is precluded in the reaction scheme making the methodology even more interesting from the environmental point of view [4]. As a realization grows that organic molecules not only have ease of manipulation and "green" advantage but can also be very efficient catalysts, asymmetric organocatalysis may begin to catch up with spectacular advancements of enantioselective transition metal catalysis.

The tremendous development of Aldol reactions since 1872 has provided an array of synthetic information advancing the construction of welldefined asymmetric centers [5]. As a consequence, the synthetic utility of this reaction has grown interest in developing organocatalysts beneficial to the construction of $\beta$-hydroxy structures. Thus, as a contribution to expanding methodologies related to asymmetric Aldol reactions, we explored the utility of imidazolines in this study. Imidazolines are structural analogues of imidazoles which are widely used for chiral ligand designs, and various substituents can be introduced to the nitrogen atom for tuning steric environments and electron density [6]. There are only a few asymmetric reactions with imidazolines as an organocatalyst such as the Diels-Alder reactions (as Bronsted acid catalysts) [7] and the Morita Baylis-Hillman [8]. In this paper, we disclose the catalytic activity of several $\mathrm{C}_{2}$ symmetric, chiral 2-pyridyl-2-imidazolines organocatalysts $\mathbf{1}$ as additional organic manifolds to facilitate the construction of $\beta$ hydroxy carbonyl architectures 4 via asymmetric Aldol reactions.

\section{EXPERIMENTAL}

General. All reagents were purchased from chemical suppliers (Sigma, Merck) and were used without further purification. Colum chromatography was performed using Merck Silica Gel 60 (230-400 mesh). Visualization was carried-out with UV using Mineralight Lamp Multiband UV at $254 \mathrm{~nm}$ and $366 \mathrm{~nm}$ and vanillinsulfuric acid followed by heating at $100^{\circ} \mathrm{C}$ until colored spots appear. NMR analysis was performed in Bruker AMX 300 MHz. Samples for NMR were dissolved in $\mathrm{CDCl}_{3}$ with TMS as internal standard. Experiments include routine ${ }^{1} \mathrm{H}$ and ${ }^{13} \mathrm{C}$.

General procedure for the synthesis of imidazoline organocatalysts 1a-1d. To a solution of 2-pyridine carbaldehyde (picolinaldehyde) or 2,6 pyridine dicarbaldehyde 2 (1 equiv) in $t$-butyl alcohol $(18.5 \mathrm{~mL}$ per $1.85 \mathrm{mmol}$ ) was added diamine derivative 3 (1.1 or 2.2 equiv). The obtained mixture was stirred at room temperature and $\mathrm{K}_{2} \mathrm{CO}_{3}$ (3 or 6 equiv) and $\mathrm{I}_{2}(1.25$ or 2.50 equiv $)$ were added and stirred at $70^{\circ} \mathrm{C}$ for $4 \mathrm{~h}$. The mixture was quenched with saturated $\mathrm{Na}_{2} \mathrm{SO}_{3}$ until iodine disappeared and extracted with DCM $(3 \times)$. The organic layer was washed with saturated $\mathrm{NaHCO}_{3}$ and dried over anhydrous $\mathrm{Na}_{2} \mathrm{SO}_{4}$. After filtration, the solvent was evaporated and chromatographed using 9:1 DCM-methanol to obtain $\mathbf{1}$.

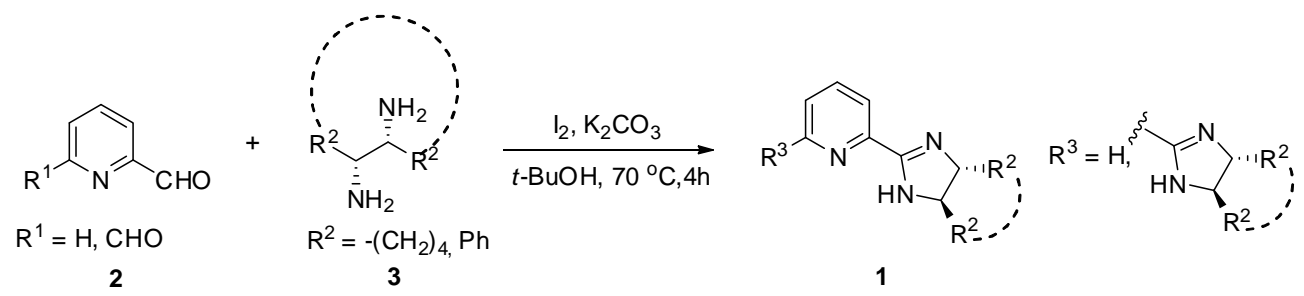

Scheme 1. General scheme for the synthesis of picolinylimidazoline organocatalysts $\mathbf{1}$. 


\section{Enantioselective chiral 2-pyridyl-2-imidazoline}

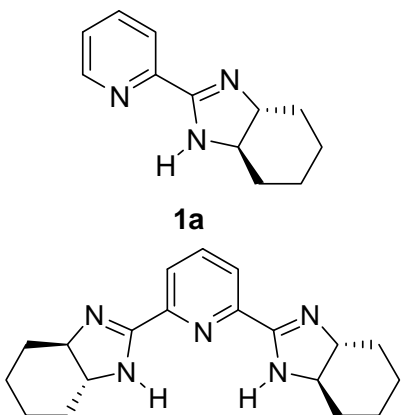

$1 b$<smiles>c1ccc([C@@H]2N=C(c3ccccn3)N[C@H]2c2ccccc2)cc1</smiles><smiles>c1ccc([C@@H]2N=C(c3cccc(C4=N[C@H](c5ccccc5)[C@H](c5ccccc5)N4)n3)N[C@H]2c2ccccc2)cc1</smiles>

$1 d$
${ }^{1} \mathrm{H}$ NMR $\delta\left(300 \mathrm{MHz}, \mathrm{CDCl}_{3}\right) 8.51(\mathrm{~d}, 1 \mathrm{H}, J=4.6 \mathrm{~Hz}, \mathrm{Py}-6-\mathrm{H})$, 8.13 (d, $1 \mathrm{H}, J=7.8 \mathrm{~Hz}$, Py- 3, 3-H), 7.83-7.75 (m, 1H, Py), 7.29 (m, 1H, Py), 3.20-3.09 (br m, 2H, 2 x CH) 2.31-2.19 (br, m, 2H, $\mathrm{CH}_{2}$ ), 1.83-1.72 (br m, $2 \mathrm{H}, \mathrm{CH}_{2}$ ), 1.58-1.45 (br m, $2 \mathrm{H}, \mathrm{CH}_{2}$ ), 1.36- 1.24 (br m, $2 \mathrm{H}, \mathrm{CH}) .{ }^{13} \mathrm{C}-\mathrm{NMR} \delta\left(100 \mathrm{MHz}, \mathrm{CDCl}_{3}\right)$ $165.3,148.8,148.5,136.7,125.3,122.2,30.8(\mathrm{CH}), 25.0\left(\mathrm{CH}_{2}\right)$ [9].

${ }^{1} \mathrm{H}$ NMR (300 MHz, $\left.\mathrm{CDCl}_{3}\right)$ 1.34, m, 4H), $1.55(\mathrm{~m}, 4 \mathrm{H}), 1.83$ (m, 4H), $2.28(\mathrm{~m}, 4 \mathrm{H}), 3.36$ (brm, 4H) $7.81(\mathrm{t}, J=7.3 \mathrm{~Hz}, 1 \mathrm{H})$, $7.21(\mathrm{~d}, J=7.3 \mathrm{~Hz}, 2 \mathrm{H}) ;{ }^{13} \mathrm{C}$ NMR $\left(100 \mathrm{MHz}, \mathrm{CDCl}_{3}\right): 21.6$, $22.9,30.7,23.5,137.3,147.8,164.6[9]$.

${ }^{1} \mathrm{H} \mathrm{NMR}\left(300 \mathrm{MHz}, \mathrm{CDCl}_{3}\right): \delta 4.82(\mathrm{~d}, J=7.9 \mathrm{~Hz}, 1 \mathrm{H}), 5.13(\mathrm{~d}$, $J=8.4 \mathrm{~Hz}, 1 \mathrm{H}), 6.54(\mathrm{~s}, 1 \mathrm{H}), 7.27-7.45(\mathrm{~m}, 11 \mathrm{H}$, aromatic), 7.82 (ddd, $J=1.7,7.7,7.7 \mathrm{~Hz}, 1 \mathrm{H}$, aromatic), 8.31-8.35 (m, $1 \mathrm{H}$, aromatic), 8.62-8.65 (m, $1 \mathrm{H}$, aromatic); ${ }^{13} \mathrm{C}$ NMR $(125 \mathrm{MHz}$, $\left.\mathrm{CDCl}_{3}\right): \delta 77.2,122.7,125.3,126.0-128.6(\mathrm{Ph}), 136.6,143.2$, $148.4,148.7,162.5$ [10].

${ }^{1} \mathrm{H}$ NMR (300 MHz, $\left.\mathrm{CDCl}_{3}\right): 4.90$, bs, 4H), 7.22-7.31 (m, 20H), $7.89(\mathrm{t}, J=7.8 \mathrm{~Hz}, 1 \mathrm{H}), 8.46(\mathrm{~d}, J=7.8,2 \mathrm{H}) ;{ }^{13} \mathrm{C} \mathrm{NMR}(100$ $\left.\mathrm{MHz}, \mathrm{CDCl}_{3}\right)$ 74.9, 125.5, 127.1, 128.3, 129.2, 136.1, 138.2, $142.9,162.1[11]$.
Representative procedure for the Aldol reaction. To a mixture of imidazoline catalyst 1 (10 mole \%) and 4-nitrobenzaldehyde (1 equiv) in brine ( $1 \mathrm{~mL}$ per $0.074 \mathrm{mmol}$ ) was added acetone $(0.25 \mathrm{~mL}$ per $0.074 \mathrm{mmol})$ and stirred for $1 \mathrm{~h}$ at room temperature. Product formation was judged by TLC (9:1 DCM:MeOH). Saturated sodium bicarbonate and DCM was added and the organic layer separated and dried over anhydrous sodium sulfate. The concentrated product was column chromatographed on silica gel and eluted with 1:1 petroleum ether-ethyl acetate to give the Aldol product 4 .

Enantiomeric ratio (er) determination. Enantiomeric ratio was determined on a Shimadzu Prominence LC-20AT Pump equipped with Shimadzu SPD-10AV. Flow rate was $1.0 \mathrm{~mL} /$ min and the pump pressure was 664 psi. UV-VIS Spectrophotometric Detector was set at $254 \mathrm{~nm}$ and connected to a Shimadzu Communication Bus Module CBM-102, with a CLASS GC-10 Workstation. Separation was done on a chiral column Lux 5u Cellulose-2 $(250 \times 4.6 \mathrm{~mm})$. Solvent systems was 70:30 n-hexane/ isopropyl alcohol.

\section{RESUlTS AND DISCUSSION}

Pyridylimidazoline organocatalysts (1a-1d) were prepared through iodine-promoted condensation and oxidative cyclization from commercially available picolinaldehydes, 2 (pyridine-2-carbaldehyde, 2a or pyridine-2,6- 
dicarbaldehyde, $\mathbf{2 b}$ and) and chiral diamines, 3 $(1 R, 2 R$-cyclohexane diamine or 3a $1 R, 2 R-1,2$ diphenyl-1,2-ethylenediamine, 3b) in excellent yields (Scheme 2).

The synthesis of picolinylimidazolines $\mathbf{1}$ in general follows a mechanism (Scheme 3 ) which proceeds initially by condensation of an aldehyde with a diamine to afford imine intermediate 5 which in equilibrium, cyclizes to dihydroimidazoline 6 [12]. Iodine-promoted oxidative addition followed by elimination thus proceeds to the target picolinylimidazolines $\mathbf{1}$.

The catalytic activity of 2-pyridyl-2-imidazolines 1a-1d was evaluated in the direct enantioselective Aldol reaction of 4nitrobenzaldehyde and acetone. Optimization of catalyst loading, temperature and solvent were done and the results are presented in Table 1. The best catalyst was found to be the $C_{2}$ symmetric 1c (Entry 10) giving the highest yield (78\%) and excellent enantiomeric ratio (99:1) with high preference and selectivity to the formation of the $R$-configurated $\beta$-hydroxy Aldol product (4a).

Brine and a $10 \mathrm{~mol} \%$ catalyst $1 \mathrm{c}$ were observed to be the best solvent and catalyst loading. No significant improvement in yield was obtained when the catalyst loading was increased to 15 mol\% while a longer reaction time was required when a $5 \mathrm{~mol} \%$ catalyst was used. Lowering the temperature to $0^{\circ} \mathrm{C}$ did not improve the yield and enantioselectivity.

The addition of $\mathrm{NaCl}$ in the reaction promotes "salting in" effect accelerating the
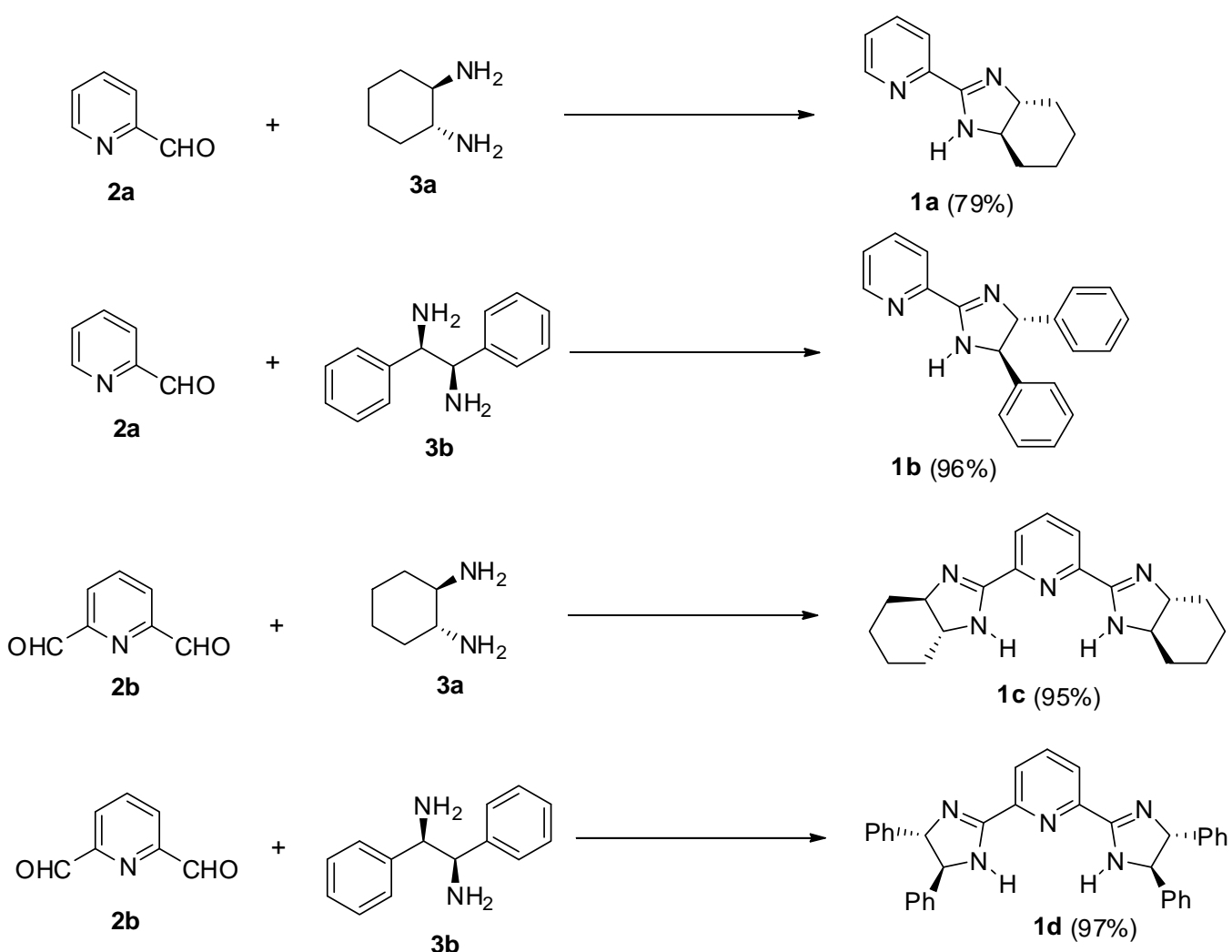

Scheme 2. Synthesis of picolinylimidazolines 1a-1d.

Reaction conditions: $\mathrm{I}_{2}$ (1.25 equiv), $\mathrm{K}_{2} \mathrm{CO}_{3}$ ( 3 equiv), $t-\mathrm{BuOH}, 70^{\circ} \mathrm{C}, 4 \mathrm{~h}$. 


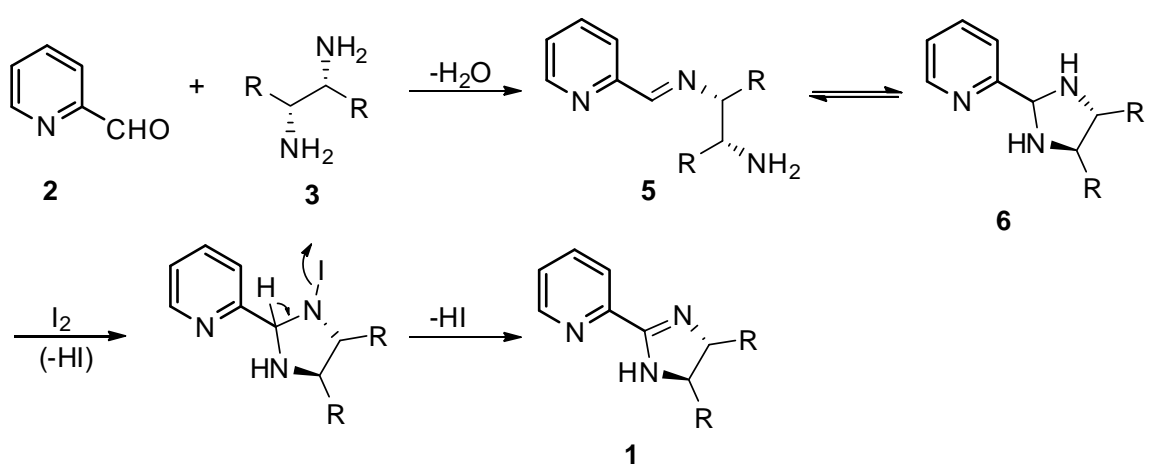

Scheme 3. Proposed mechanism en route picolinylimidazoline organocatalysts 1 .

Table 1. Optimization of organocatalyzed direct Aldol reaction conditions

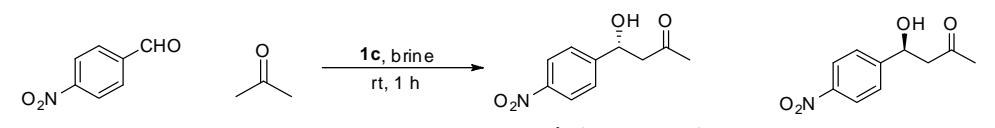

4a ( $R$ enantiomer) $\quad \mathbf{4 b}(S$ enantiomer $)$

\begin{tabular}{c|c|c|c|c|c}
\hline Entry & Solvent & Catalyst & $\begin{array}{c}\text { Catalyst loading } \\
\text { (mol \%) }\end{array}$ & $\begin{array}{c}\text { Isolated } \\
\text { Yield }\end{array}$ & er (R:S) \\
\hline 1 & $\mathrm{CHCl}_{3}$ & 1a & 20 & 59 & $79: 21$ \\
\hline 2 & $\mathrm{DCM}$ & 1a & 20 & 35 & $57: 43$ \\
\hline 3 & $\mathrm{H}_{2} \mathrm{O}$ & 1a & 20 & 70 & $91: 9$ \\
\hline 4 & Brine & 1a & 20 & 60 & $94: 6$ \\
\hline 5 & $\mathrm{CHCl}_{3}$ & 1b & 10 & 63 & $99: 1$ \\
\hline 6 & Brine & 1c & 10 & 53 & $70: 30$ \\
\hline 7 & DCM & 1c & 10 & 46 & $96: 4$ \\
\hline 8 & $\mathrm{H}_{2} \mathrm{O}$ & 1c & 10 & 65 & $91: 9$ \\
\hline 9 & Brine & 1c & 5 & 29 & $99: 1$ \\
\hline 10 & Brine & 1c & 10 & 78 & $99: 1$ \\
\hline 11 & Brine & 1c & 15 & 14 & $98: 2$ \\
\hline 12 & Brine & 1d & 10 & 58 & $94: 6$ \\
\hline
\end{tabular}

organocatalyzed Aldol reaction in water by favouring the aggregation of organic species/ intermediates in concentrated hydrophobic pockets. Very polar solutions are superior in solubilizing a polar substrate compared to less polar derivatives. Adding salt to an aqueous solution with moderate polarity decreases solubility. On the other hand, adding a (ionic) salt to solution with high polarity increases solubility provided no common ion effect is observed and a globular, micelle structure is formed in aqueous solutions. The structure of water near the head group is organized, thus, allowing weak interaction with other ions in solutions. The hydrogen bonds thus formed, are less available to interact with the soluble ion form of the salt. The same result was noted when catalyst loading was reduced down to $10 \%$ (Entry 10). In this condition, addition of brine is favoured and effective than solely employing water as the solvent.

Addition of selected organic acids did not improve yield and enantioselectivity (Table 2). This implies that a Bronsted acid-mediated mechanism may not be involved in the catalytic pathways. Instead, a Bronsted-base catalyzed generation of enol and H-bonding stabilized 
intermediate is observed. In addition to these acid additive serving as Bronsted co-catalyst, the results obtained by Masse and co-workers [13] agree with our findings that improved catalytic performance can only be obtained in polar aprotic solvent (for example DMSO) but not in polar, protic solvents such as water. In all cases, the additive weak organic acids were afforded in moderate yield but with high enantioselectivities up to $98 \%$ (Table 2). This indicates that in the course of the reaction development, the catalyst additive associates with the reactants similar to that of micelles.

To determine the scope of the reaction, various benzaldehyde derivatives were also screened (Table 3). Aldol reaction of aromatic aldehydes 7 with acetone 8 afforded the $\beta$-hydroxy derivatives $\mathbf{4}$ in moderate to excellent yields. The 4-(m-chlorophenyl)-4-hydroxy-2-butanone $\mathbf{4 e}$ was obtained with the high yield, with almost no self-aldolization product and excellent enantioselectivity (Entry 5). Significant decrease in yield was noted when the substituent is a bromine atom at C-4 (Entry 6, 4f) while a drop in

Table 2. Effect of Bronsted acid additives

\begin{tabular}{c|c|c|c}
\hline Entry & Additive & Isolated Yield & er (R:S) \\
\hline 1 & $L$-Tartaric acid & 34 & $98: 2$ \\
\hline 2 & $L$-Citric acid & 17 & $95: 5$ \\
\hline 3 & Benzoic acid & 33 & $75: 25$ \\
\hline
\end{tabular}

enantiomeric ratio was observed when the electrophile is dimethoxylated at C-3 and C-4 for 4g. (Entry 7) Sluggish reaction period was also observed when the substituent is a methyl group at C-4 (Entry 4 for 4d). Finally, among the electron withdrawing substituents at $\mathrm{C}-4$, the presence of a nitrile group (Entry 2) effected a drop in the yield of the Aldol product $4 \mathbf{b}$ compared to the 4nitro derivative $\mathbf{4 a}$ (Entry 1).

Based on the results obtained where a high selectivity towards the $R$ enantiomer is favoured, a hydrogen-bonding - stabilized transition state is proposed whereby the incipient bond is formed between the $R e$ face of the benzaldehyde electrophile and the Si face of the enol nucleophile.

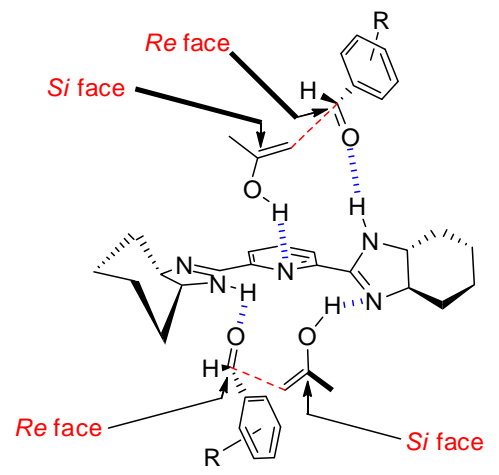

Figure 1. Proposed transition state model for the enantioselective, direct aldol reaction with 1c.

Table 3. Scope of the organocatalyzed Aldol reaction with 1c

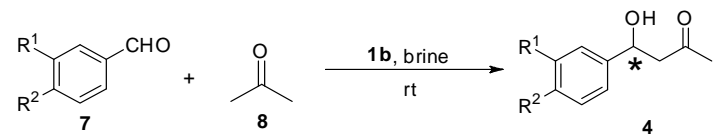

\begin{tabular}{c|c|c|c|c|c|c}
\hline Entry & $\mathbf{R}^{\mathbf{1}}$ & $\mathbf{R}^{\mathbf{2}}$ & Product 4 & $\begin{array}{c}\text { Reaction } \\
\text { Time (h) }\end{array}$ & $\begin{array}{c}\text { Isolated } \\
\text { Yield }\end{array}$ & er (R:S) \\
\hline 1 & $\mathrm{H}$ & $\mathrm{NO}_{2}$ & $\mathbf{4 a}$ & 1 & 78 & $99: 1$ \\
\hline 2 & $\mathrm{H}$ & $\mathrm{CN}$ & $\mathbf{4 b}$ & 2.5 & 26 & $99: 1$ \\
\hline 3 & $\mathrm{H}$ & $\mathrm{OMe}$ & $\mathbf{4 c}$ & 3 & 99 & $99: 1$ \\
\hline 4 & $\mathrm{H}$ & $\mathrm{Me}$ & $\mathbf{4 d}$ & 24 & 37 & $99: 1$ \\
\hline 5 & $\mathrm{H}$ & $\mathrm{Cl}$ & $\mathbf{4 e}$ & 2 & 98 & $>99$ \\
\hline 6 & $\mathrm{H}$ & $\mathrm{Br}$ & $\mathbf{4 f}$ & 2 & 9 & $99: 1$ \\
\hline 7 & $\mathrm{OMe}$ & $\mathrm{OMe}$ & $\mathbf{4 g}$ & 2.5 & 50 & $51: 49$ \\
\hline
\end{tabular}




\section{Enantioselective chiral 2-pyridyl-2-imidazoline}

Four chiral picolinylimidazoline organocatalysts were successfully synthesized and employed as catalysts for the direct asymmetric Aldol reaction of benzaldehydes and acetone. The $C_{2}$ symmetric imidazoline 1c was observed to be the best organocatalyst with brine as the reaction solvent and $10 \mathrm{~mol} \%$ as the best catalyst loading at room temperature. Moderate to excellent amounts of the $\beta$-hydroxy Aldol product 4 were obtained in excellent enantioselectivity favoring the $R$ enantiomer. Our study provides a green, easy approach and straightforward enantioselective access to $\beta$ hydroxy ketone motifs.

\section{REFERENCES}

[1] Bhanushali M \& Zhao C-G. Developing Novel Organocatalyzed Aldol Reactions for the Enantioselective Synthesis of Biologically Active Molecules. Synthesis 2011; 12:1815-1830.

[2] Liu WJ \& Gong LZ. Asymmetric Organocatalysis. Topics in Organometallic Chemistry 2011; 36:153-206.

[3] MacMillan DWC. The Advent and Development of Organocatalysis. Nature 2008; 455:304-308.

[4] Alemán J \& Cabrera S. Applications of Asymmetric Organocatalysis in Medicinal Chemistry. Chemical Society Reviews 2013; 42:774-793.

[5] Denmark SE, Heemstra JR, \& Beutner GL. Catalytic, Enantioselective, Vinylogous Aldol Reactions. Angewandte Chemie-International Edition 2005; 44:4682-4698.

[6] Liu H \& Du D-M. Recent Advances in the Synthesis of 2-Imidazolines and their Applications in Homogeneous Catalysis. Advanced Synthesis \& Catalysis 2009; 351:489-519.
[7] Davenport AJ, Davies DL, Fawcetta J, \& Russella DR. Chiral Pyridylimidazolines: Synthesis, Arene Ruthenium Complexes and Application in Asymmetric Catalysis. Journal of the Chemical Society, Perkin Transactions 2001; 1:15001503.

[8] Xu J, Guan Y, Yang S, Ng Y, Peh G, \& Tan CH. Asymmetric Baylis-Hillman reactions promoted by chiral imidazolines. Chemistry - An Asian Journal 2006; 1:724-729.

[9] Tydlitát J, Bureš F, Kulhánek J, \& Rù•ièkab A. 1,2-Disubstituted Hexahydro-1H-benzo[d] imidazoles: Synthesis, Characterization, and Stability. Synthesis 2010; 22:3934-3940.

[10] Fruhstorfer W \& Muller-Calgan H (E. Merck Aktiengesellschaft, Darmstadt, Germany) Imidazoline Derivatives. US Patent 3, 147, 275 (September 1, 1964).

[11] Bhor S, Anilkumar G, Tse MK, Klawonn M, Doebler C, Bitterlich B, Grotevendt A, \& Beller M. Synthesis of a New Chiral N,N,N-Tridentate Pyridine bisimidazoline Ligand Library and Its Application in Ru-Catalyzed Asymmetric Epoxidation. Organic Letters 2005; 7:3393-3396.

[12] Ishihara M \& Togo H. An Efficient Preparation of 2-Imidazolines and Imidazoles from Aldehydes with Molecular lodine and (Diacetoxyiodo) benzene. Synlett 2006; 37:227-230.

[13] Delaney JP \& Henderson LC. Investigating Ionic Effects Applied to Water Based Organocatalysed Aldol Reactions. International Journal of Molecular Sciences 2011; 12:9083-9094.

[14] Mase N, Nakai Y, Ohara N, Yoda H, Takabe K, Tanaka F, \& Barbas CFJ III. Investigating Ionic Effects Applied to Water Based Organocatalysed Aldol Reactions. Journal of the American Chemical Society 2006; 128:734-735. 